\title{
Caractérisation endoscopique des milieux granulaires couplée à l'essai de pénétration
}

P. BREUL

SOL SOLUTION

ZAC des portes de Riom

63200 Riom

\section{R. GOURVES}

LERMES/CUSI

Campus universitaire

des Cézeaux:

BP 206

63174 Aubière Cedex

Cet article présente une nouvelle technique de caractérisation in situ des milieux granulaires utilisant I'endoscopie et l'analyse d'images, La première partie présente les objectifs de cette technique et décrit la méthode d'investigation utilisée, La seconde partie est consacrée à l'ensemble des caractéristiques extraites automatiquement à partir des images. On śattarde plus particulièrement sur l'analyse de la texture des images. sur l'étude de la granulométrie des matériaux, enfin sur l'étude de l'angularité et de la colorimétrie. Cette partie se termine sur la présentation d'une démarche générale d'analyse divisée en trois niveaux. Enfin, la dernière partie présente sur un exemple d'application réelle de caractérisation des remblais : l'utilisation de cette technique met en évidence l'intérêt du couplaqe avec les essais de pénétration.

\section{Characterisation of granular materials by using endoscopy and penetration test}

NDLE : Les discussions sur cet article sont acceptées dans un délai de trois mois après sa parution.
This paper deals with a new technique of a in situ granular medium chatacterisation using encloscopy and images analysis. The first part sets out techniques aims and describes the investigation method. The second part is devored to the characteristics automatically extracted from images. We are studying more precisely images texture, materials particles size analysis, angularity and colorimetric characteristics. At least we proposed a general approach of anafysis divided in three stages The last part deals with a real application of embankment characterisation by using the technique. This example underlines the coupling interest of this technlque with the penetration tests: 


\section{Introduction}

Le comportement des milieux granulaires est étroitement lié à leur microstructure. Une description complète de cette microstructure permet une meilleure estimation du comportement global du matériau. Mais l'obtention des paramètres décrivant la microstructure est impossible in situ et délicate de manière expérimentale sur des matériaux réels. C'est pourquoi, la mécanique des milieux continus, qui permet d'obtenir les caractéristiques globales des matériaux à partir d'essais réalisés en laboratoire ou in situ, est largement utilisée à theure actuelle.

Les essais en laboratoire permettent une identification précise des matériaux du point de vue mécanique et physique ; ils sont relativement bien maitrisés. Mais IIs sont parfois longs, chers et pratiqués sur des échantillons remaniés donc partiellement représentatifs de l'état réel du matériau. C'est pourquoi on assiste à un développement des essais in situ qui, bien que porteurs de peu d'information (un ou deux paramètres mécaniques), ont l'avantage de sonder le milieu dans son état naturel et de pouvoir être multipliés et couplés avec d'autres essais, ce qui permet ainsi une bonne caractérisation et une estimation de la variabilité du sol.

L'essai de pénétration est l'un des essais in situ classique utilisé en reconnaissance géotechnique et en contróle des remblais. Il fournit des informations sur l'état de serrage du matériau en fonction de la profondeur. Mais cet essai est " aveugle n. Aussi l'utilisation de l'endoscopie pour récolter des images et de l'analyse d'images pour étudier les matériaux gramulaires in situ d'une manière peu traumatisante, peut permettre de retrouver les caractéristiques physiques des matériaux.

Cet article présente le procédé de prise d'images in situ des matériaux ainsi que les différentes caractéristiques extraites automatiquement à partir des images récoltées. Enfin un exemple pratique de caractérisation de remblai permet de mettre en évidence l'intérêt du couplage de cette technique avec les essais de pénétration.

\section{2}

\section{Le procédé de prise d'images}

\section{1}

\section{Les objectifs du procédé}

Le but du procédé est de récolter des images de milieux granulaires in situ, dans la cavité laissée par un essai de pénétration de 16 ou $23 \mathrm{~mm}$ de diamètre (lors de notre travail nous avons essentiellement utilisé lè pénétromètre dynamique de type PANDA (Gourves, 1991 ; Zhou, 1997). Étant donné la nature du milieu ausculté, le procédé doit d'être robuste (milieu effondrable, abrasif, humide...). Pour avoir un intérêt pratique, il doit être peu encombrant, facile à manipuler et d'un temps court de mise en œuvre et d'intervention. Pour permettre l'observation ou le contróle d'ouvrages en service, il doit ètre peu traumatisant. Enfin, les images recueillies doivent être d'une qualité suffisante pour permettre leur exploitation ultérieure.

\section{Dispositif expérimental et méthode d'investigation}

A la suite d'un essai au pénétromètre dynamique, on utilise la cavité laissée par l'essai (diamètre de la cavité : $16 \mathrm{~mm}$ ) pour introduire un endoscope comprenant un capteur à transfert de charge (CCD) et un éclairage par fibre optique, et pouvoir ainsi récolter des images du sol tout le long de la cavité. Afin d'obtenir des images de la meilleure qualité possible, un dispositif permettant de maintenir et de stabiliser l'endoscope lors de la prise d'image a été mis en place (Fig. 1). L'utilisation d'un tube percé de fenêtres dont l'écartement des bords est défini avec précision permet de connaitre, pour chaque image, le grossissement avec lequel on travaille. Les images, une fois récoltées, sont stockées numériquement pour ètre traitées et analysées ultérieureinent.

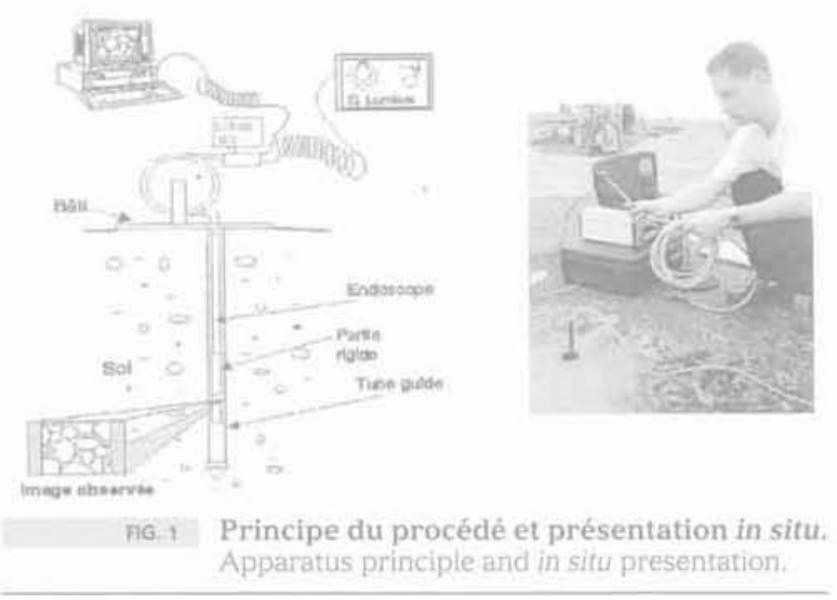

\section{Images obtenues}

Un exemple d'images obtenues par ce procèdé est donné ci-dessous (Fig, 2). Les images acquises sont en couleur, de taille $640^{*} 480$ pixels. La partie de l'image contenant réellement le matériau granulaire observé correspond à un carré de $5 \mathrm{~mm}$ de côté. Deux phénomènes influent sur la qualité de ces images. Tout d'abord IThomogénéité de l'éclairage, qui est souvent plus fort au centre de l'image qu'en périphèrie et aussi la texture et le degré d'humidité des matériaux qui sont susceptibles de favoriser la présence de zones de reflet dans l'image.

Ce dispositif permet d'ausculter les sols jusqu'à une profondeur de $7 \mathrm{~m}$. Les avantages d'un tel procédé sont de pouvoir effectuer un grand nombre d'essais rapidement et sans traumatisme. Du fait du champ de vision que procure l'endoscope, on travaille pour le moment sur des matériaux dont le diamètre des grains varie de 0 à $5 \mathrm{~mm}$. Le passage à l'étude de matériaux plus grossiers fait l'objet des recherches futures.

\section{3}

\section{Différentes caractéristiques étudiées à partir des images}

Parmi les paramètres locaux caractérisant les sols, la texture, la granulométrie, l'angularité et la couleur 


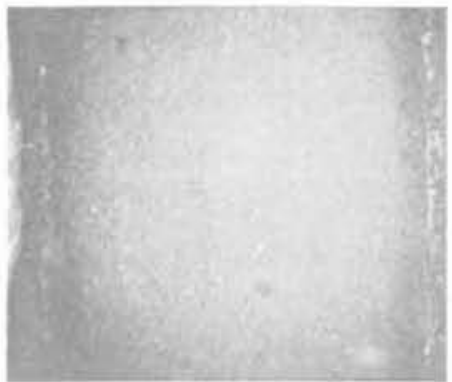

Limon (Arles - 0/0,5 - AI)

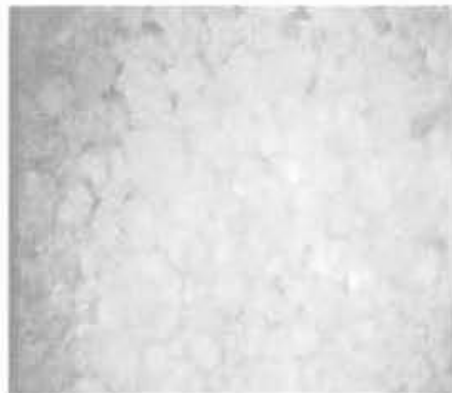

Sable gréseux ( 0/2 - B5)

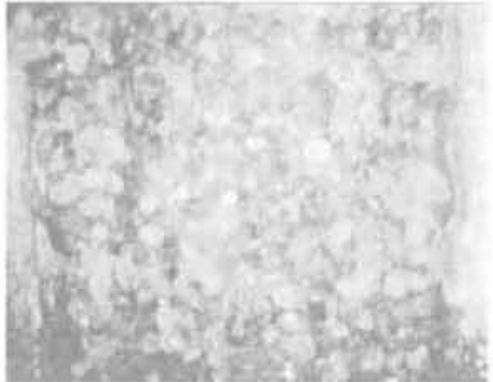

Sable (Allier - 0,1/0,315 - B5)

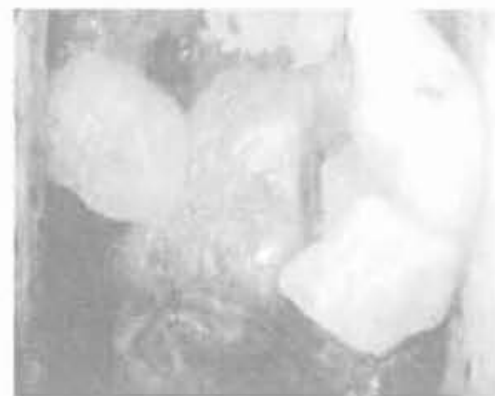

Silico-calcaire ( 1/5 - DC1)

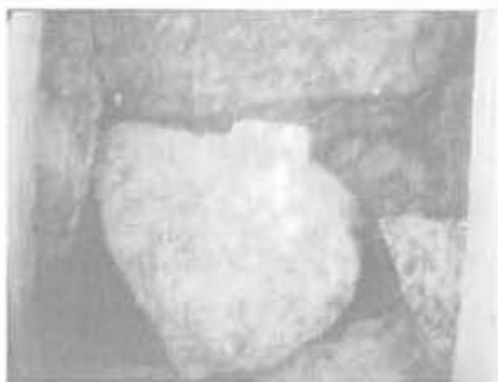

Basalte (2/8 - DC3)

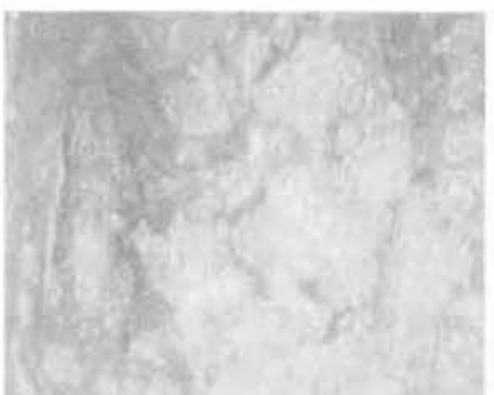

Pouzzolane (D1)

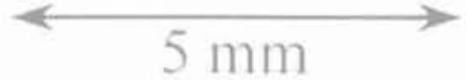

मG. 2. Exemples d'images obtenues avec le procédé (classification GTR). Images obtained by using this apparatus (GIR classification).

des matériaux ont été étudiées. La granulométrie est sans conteste le paramètre le plus important, pour l'identification, la classification et la prévision du comportement d'un sol.

En effet, la plupart des classifications de sols utilisent ce parametre et notamment la classification GTR du Guide pour les terrassements routiers (SETRA, 1994). qui sert de référence en France pour tout ce qui concerne les matériaux cie remblais, L'angularité et la couleur des particules composant le sol sont des caractéristiques secondaires mais elles permettent de différencier les couches de matériaux entre elles et d'obtenir rapidement des informations sur l'origine des matériaux.

\section{1}

\section{Caractérisation de la texture}

L'analyse de la texture des images a deux objectifs. Premièrement dissocier les matériaux fins pour lesquels une analyse granulométrique par analyse d'images n'est pas envisageable, des matériaux plus grenus sur lesquels cette analyse est possible et deuxièmement caractériser les matériaux fins.

Parmi les trois principales approches utilisées (statistique, structurelle et spectrale) pour caractériser la texture d'une image (Gonzalez, 1992), on a adopté les approches statistique et spectrale (Breul, 1999). L'approche spectrale est basée sur l'étude de la trans- formée de Fourier F(u, v) de l'image où u et v représentent les variables de fréquence, Raschke (1996) a montré que la texture d'une image de sol peut être caracté. risée par le spectre de puissance $\mathrm{P}(\mathrm{u}, \mathrm{v})=|\mathrm{F}(\mathrm{u}, \mathrm{v})|$ ou de manière plus simple par le moment normalisé (Mi) du logarithme normalisé des valeurs du spectre de puissance, défini par:

$$
M i=\frac{\sum_{n=0}^{N} n^{\prime} P^{n}(n)}{\sum_{n=0}^{N} n^{\prime}}
$$

oú : $i=$ ordre du moment :

$\mathrm{N}=$ nombre total de termes de $\mathrm{P}^{*}(\mathrm{n})$ considéré :

$\mathrm{P}^{\prime \prime}(\mathrm{n})=$ logarithme normalisé du spectre de puissance.

Des études non fournies dans cet article (Breul. 1999) ont montré que, sur les images, l'utilisation d'un moment d'ordre 3 permettait la meilleure discrimination des différentes textures de sol. Une étude sur différents matériaux a été réalisée, afin d'évaluer l'influence de la granulométrie des matériaux sur la valeur de M3. La figure 3 fournit les valeurs moyennes de M3 pour chaque image (valeur brute), ainsi que la moyenne de ces valeurs pour chaque matériau (valeur moyerne). Cette étude permet de mettre en évidence la possibilité de dissocier les matériaux grenus des matériaux fins. En effet, plus un matériau est grenu. plus la valeur moyenne des moments normalisés est faible et plus les valeurs brutes sont dispersées. 


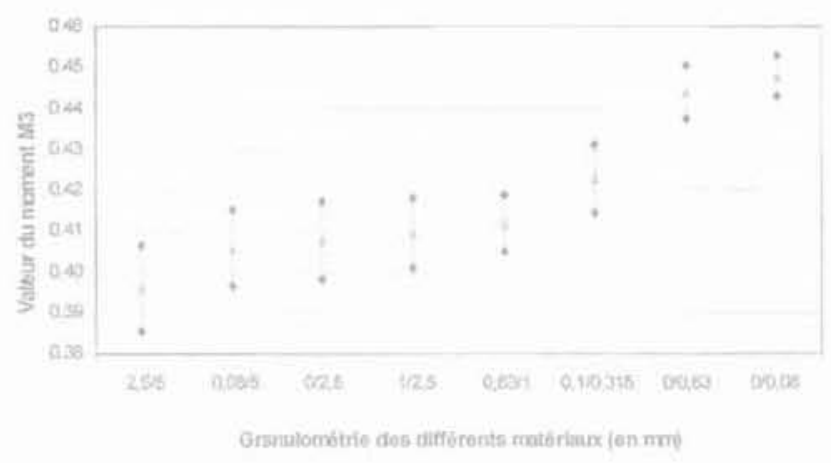

FG. 3 Variation des valeurs du moment M3 pour différents matériaux (classés du plus grossier au plus fin) (intervalle de confiance à $95 \%$ ).

Vartation of $M 33$ values for cilferent materials (classifled from the coarsest to the finest) (confidence interval at $95 \%$ )

Une seconde étude a été menée afin de voir l'influence de la proportion de particules fines (particules de taille inférieure à $80 \mu \mathrm{m}$ ) dans un matériau, sur la valeur du moment M3. Deux zones peuvent étre mises en évidence (Fig. 4). Jusqu'à un pourcentage de fines particules de l'ordre de 30 à $40 \%$, plus la proportion de particules fines augmente, plus le moment normalisé est important, mais reste en dessous de la valeur 0,43 . Par contre, au-dessus d'une proportion de $40 \%$ de particules fines, on ne constate pratiquement aucune variation de texture, et le moment M3 est toujours supérieur à 0,43 .

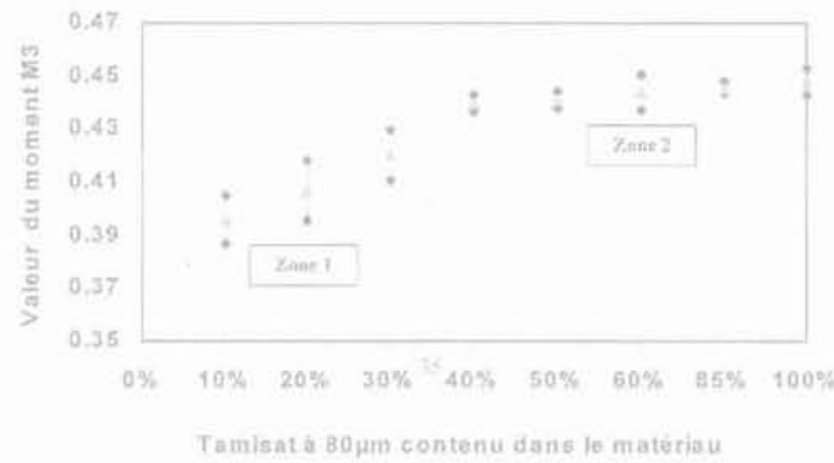

FG. 4 Variation des valeurs du moment Mi en fonction de la proportion de particules inférieures à $80 \mu \mathrm{m}$ contenues dans le matériau.

Variation of Mif values taking into account the $80 \mu \mathrm{m}$ finer particles percentage.

Cette dernière remarque est intéressante, car le seuil de $35 \%$ de particules inférieures à $80 \mu \mathrm{m}$ dans un matériau marque la limite dans la classification GTR, entre les matériaux classès A (matériaux très fins et donc très sensibles à l'eau) et les autres matériaux. Une valeur de M3 supérieure à 0,43 permet donc de distinguer les matériaux très fins des autres matériaux.

L'observation des images de matériaux comportant une proportion de particules fines inférieure à $10 \%$. nous permet de dire qu'en dessous de cette valeur, il est possible d'effectuer une analyse granulométrique sur les images. Il est donc intéressant de trouver un paramètre permettant de séparer les matériaux sur lesquels sera effectuée une analyse granulométrique, des autres (ceux dont la proportion de fines particules est trop importante). L'analyse spectrale n'apporte pas de réponse pour ce genre de matériaux car les valeurs de M3 accusent une dispersion trop importante d'une image à l'autre (Fig. 3), Aussi, une approche statisticue basée sur l'étude de l'histogramme des images et plus particulièrement sur l'écart-type el le coefficient de variation des niveaux de gris est utilisée pour clistinguer ces materiaux (Fig. 5).

L'étude statistique permet de voir que dans nos conditions de prise d'images, les images de matériaux dont la proportion de particules fines est inferieure à $10 \%$, possèdent un écart-type supérieur à 40 niveaux de gris et un coefficient de variation supérieur à $25 \%$. En effet, plus un matêriau est grenu, plus il y a de variations importantes de niveaux de gris sur son image, du fait de la différence de matière entre les grains, et de la présence de contours plus marqués. Ces deux parametres permettent donc de dissocier les matériaux sur lesquels une analyse granulométrique est possible.
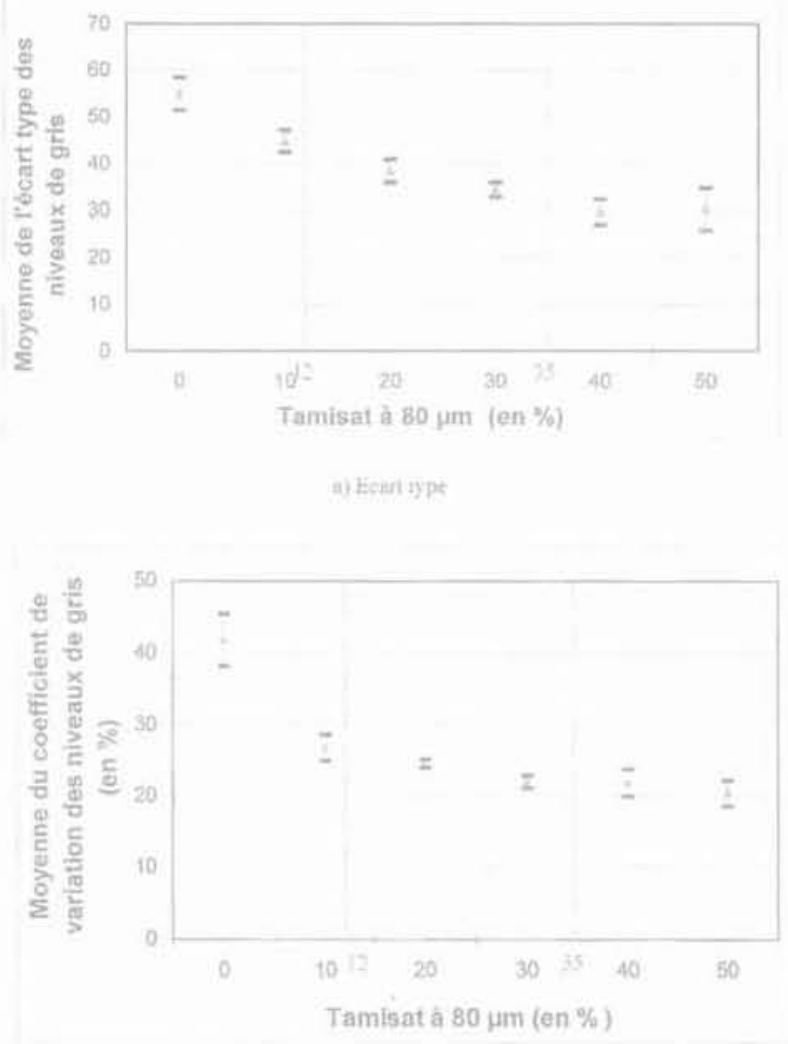

He.s Variation de l'écart-type et du coefficient de variation de l'histogramme en niveau de gris en fonction de la proportion de particules inférieures à $80 \mu \mathrm{m}$ contenues dans le matériau (intervalle de confiance à $95 \%)$.

Influence of the $80 \mu \mathrm{m}$ finer particles percentage on the image standard deviation (a) and grey level variation coefficlent (b) (confidence interval at $95 \%$. 


\section{Caractérisation granulométrique}

Préalablement à l'exécution de l'analyse granulométrique proprement dite, il est nécessaire d"appliquer un traitement aux images afin d'en améliorer les caractéristicues et de faire ressortir les centres d'intéréts. En ce quif nous concerne, le but principal du traitement est d'extraire un maximum de contours des particules de matériaux se trouvant dans l'image en utilisant des outils de traitement classiques (filtre gaussien, transformée chapeau haut de forme, extraction de contours...). Une fois le traitement terminé, l'analyse granulométrique est menée. Les méthodes classiques d'analyse grain par grain iméthode des érodés ultimes, du squelette des vides, de la ligne de partage des eaux...) (Gonzalez. 1992) n'ayant pas donné de résultats satisfaisants, aussi bien du poirit de vue de la déconnexion des particules que du temps d'analyse, les recherches ont été orientées vers les méthodes d'anaiyse ensembliste, qui examinent l'image dans sa globalité.

Deux methodes ont êté utilisées. L'analyse pat ouverture morphologique (Coster, 1985), en utilisant des éléments structurants carrés, et une méthode basée sur l'étude des traversées de I'image (Fig. 6). L'analyse par ouverture si elle donne des résultats satisfaisants à l'inconvénient d'être moins rapide que l'analyse par traversées et surtout plus sensible aux chevauchements des particules.

La comparaison des résultats obtenus par ces deux méthodes (Fig. 6) et par tamisage manuel « classique ») montre que les granulométries par analyse d'images permettent d'obtenir le diamètre des plus gros grains composant le matériau, mais surestiment toujours la proportion de fines particules présentes dans le matériau. Ce biais dans l'analyse est du principalement au chevauchement entre particules de l'image.

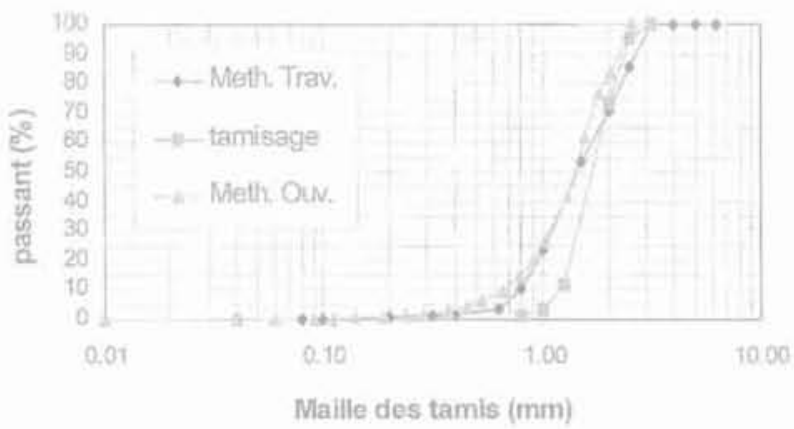

Fig. 6 Comparaison des résultats d'analyse granulométrique obtenus par analyse d'images (méthode par ouverture et par traversées) et par tamisage manuel.

Particles size distribution : comparison between manual sieving and image analysis by crossing and opening method.

Pour prendre en compte le biais introduit dans les analyses par le chevauchement des particules, deux méthodes sont utilisées. La première (Fig. 7 ) repose sur la probabilité du plan de coupure, elle consiste connaissant le diamètre $Y$ des particules, mesuré à partir de l'analyse des images, à estimer X le diamètre réel de ces particules. Pour cela à partir de la fonction de densité de $\mathrm{Y}$, il est nécessaire de déterminer par intégra- tion la fonction granulométrique en nombre des particules, puis par dérivation de cette dernière la fonction de répartition de X ou courbe granulométrique du matériati.

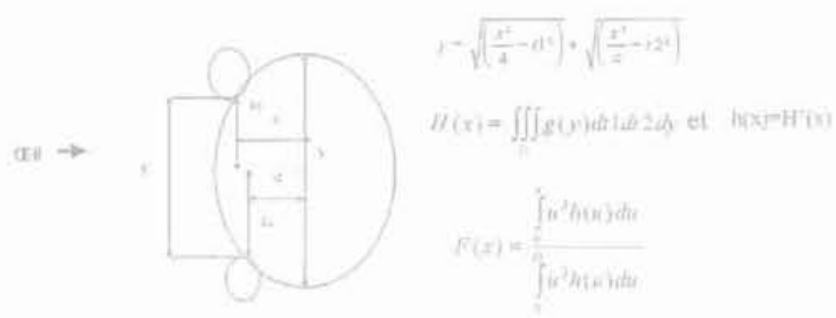

NG.7 Principe de la méthode probabiliste du plan de coupure.

Probabilistic method principle.

Cette méthode itérative reste tongue en temps d'analyse et sa précision clépend du pas choisi dans les méthodes d'intégration (en l'occurrence méthode des trapezes).

La seconde méthode mise en place est une méthode par recalage, Basée sur le fait que le biais introduit par le chevauchement des particules est fonction du diamètre des plus grosses particules composant le matériau et de l'étendue de la granulométrie du matériau. cette methode permet de recalculer la courbe granulométrique réelle du matériau analysé à partir des résultats obtenus par l'analyse d'images et du calcul du diamètre maximum et minimum du matériau.

Les résultats obtenus par cette méthode (Fig. 8) permettent de retrouver avec une bonne précision les courbes granulométriques obtenues par tamisage manuel et ce dans un temps d'analyse plus réduit.

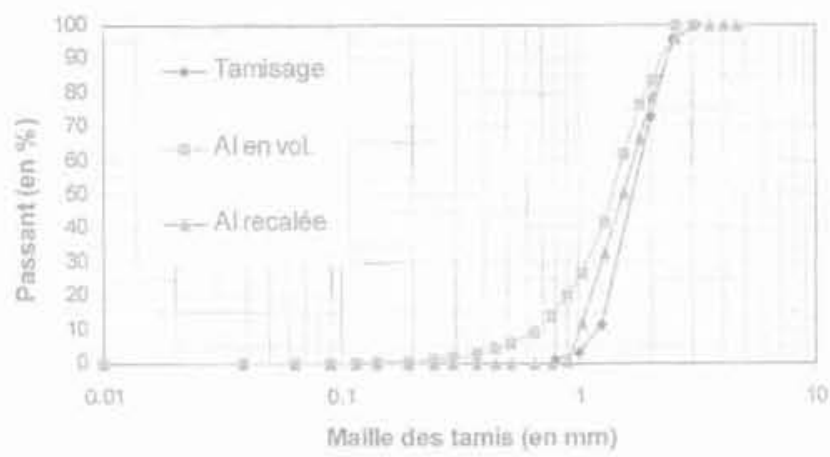

He. 8 Comparaison de l'analyse granulométrique par analyse d'images après prise en compte du chevauchement des particules avec la méthode par tamisage manuel (AI: Analyse d'Images).

Particles size analysis comparison betweet manual sieving and corrected image analysis method.

\section{3 :}

\section{Caractérisation de l'angularité}

L'angularité qui permet de prendre en compte la présence d'arêtes vives sur les particules composant un matériau, est un facteur important pour savoir si le matériau sera plus ou moins frottant. Pour décrire cet 
aspect des grains, on classe ceux-ci dans 3 familles, suivant s'ils sont roulés (grains sans arête vive, frottement faible), concassés (grains composés uniquement d'arêtes vives, frottement important) et semi-concassés (grains comportant des arêtes vives et des arêtes arrondies).

La démarche envisagée pour caractêriser l'anqularité des matériaux consiste à extraire, lors du traitement des images, des morceaux de contours de grains inclus dans l'image. Sur chaque contour airsi sélectionné, on recherche les points de discontinuité. Ces points sont définis comme étant des endroits d'un contour où l'angle $\theta$ entre les tangentes à gauche et à droite en ces points (Fig. 9a) n'appartient pas à lintervalle $180^{\circ} \pm \alpha$. L'angle $\alpha$ a été pris égal à $45^{\circ}$.

Afin de reconnaitre les contours appartenant à des particules roulées et ceux apparternant à des particules concassèes, des indices fonction du nombre de points de discontinuité et de l'angle (que font les tangentes en regard entre chaque point de cliscontinuité ont étè mis en place.
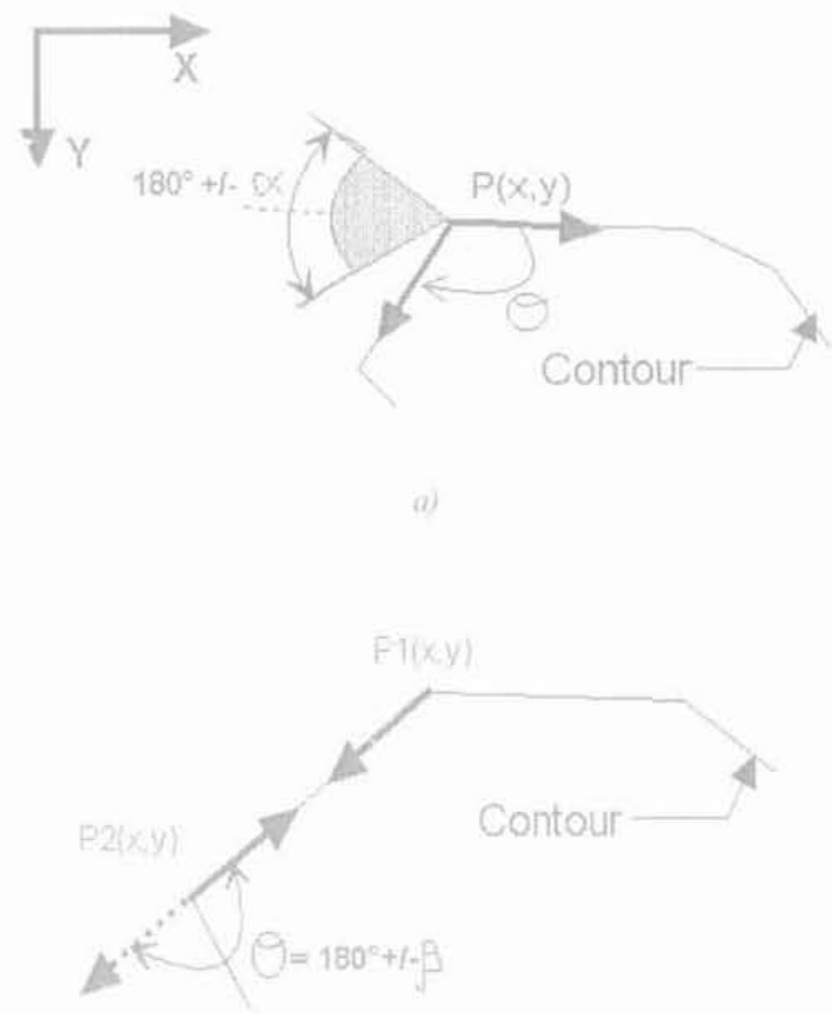

b)

FG. (a) Définition d'un point de discontinuité $(\mathrm{P}(\mathrm{x}, \mathrm{y}))$ sur un contour ; (b) Définition de l'angle $\theta$ entre les tangentes en regard de deux points de discontinuité d'un contour d'une particule concassée.

a) Disconitinuity point definition $P(x, y)$; bi $\theta$ angle definition between the tangents of two consecutive discontinuity points.

Cette méthode d'analyse de l'angularité a étẻ testée sur plusieurs matériaux naturels d'une carrière de la région lyonnaise, ainsi que sur deux matériaux non naturełs, mais pouvant ètre assimilés à un sol : des billes de verre représentant un matériau granulaire roulé et du verre pilé représentant un matériau concassé.
Les rèsultats trouvés au moyen de cette méthode sont sensibles à la grossièreté du matériau analysé et à la proportion de fines particules contenues dans ceuxci. En effet, sur les matériaux artificiels, la méthode d'analyse est très efficace car les contours des particules composant ces materiaux sont « parfaits o (faciles à détecter donc à extraire, très peu de défauts locaux dus à la texture du matériau, et morphologiquement idéaux). Par contre, pour les matériaux naturels l'efficacité de la méthode est phus relative, du fait de la complexité des images de ce type de matériaux fextraction de contours plus complexe et contours des particules de forme moins franche du fait de la presence de défauts de texture locaux). Sur des matériaux grenus et de texture peu rugueuse, la méthode permet de reconnaitre dans plus de neuf cas sur dix l'angularité du matériau. Ces statistiques diminuent notablement dès que le matériau devient plus fin ou présente une texture plus particulière comme la pouzzolane par exemple).

Enfin, à theure actuelle la notion de "semiconcassé in n'est pas détectable par l'analyse.

\section{Caractérisation colorimétrique}

Les images stockées lors de la réalisation de l'essai étant en couleur, une analyse colorimétrique a été effectuée. Le but étant de tenter de mettre en évidence qu'une étude colorimétrique des images de matériaux récoltées in situ au moyen d'un endoscope permet d'apporter des informations complémentaires à celles déjà obtenues (granulométrie et angularité). Étant donné la variété des sols existant dans la nature et. pour un même matériau (du point de vue minéralogique), la variété des teintes existantes (présence ou non d'impuretés, proportion d'impuretés, type d'impuretess), il parait impossible de retrouver la minéralogie d'un sol par cette méthode. Par coritre, il est intéressant de savoir si pour un matériau donné on arrive à retrouver une "signature in colorimétrique caractéristique. Cette signature pourrait permettre cie reconnaitre certains sols, ou d'identifier des changements de couches dans in sol.

Dans un premier temps, une étude de l'influence de l'intensité d'éclairage, de la granulométrie et de la teneur en eau sur les caractéristiques colorimétriques d'un matériau a èté effectuée, dans deux référentiels différents (référentiel Rouge Vert Bleu et H (teinte) S(saturation) I (intensité)) (Breul, 1999), Cette étude a montré que la teinte et la saturation sont les caractéristiques les plus stables. C'est pourquoi, les études suivantes ont été réalisées dans un référentiel HSI.

L'analyse effectuée a porté sur huit matériaux fins (diamètres des particules $<2$ mm) de provenance géographique et d'origine minéralogique diverses. L'analyse des graphiques présentés figure 10, montre que pour un mème matériau, les caractéristiques colorimétriques sont relativement stables sur plusieurs images. De plus, I'utilisation des paramètres H, S et I permet de dissocier la plupart des matériaux entre eux. II est à noter que le parametre “ niveau de bleu » peut aussi s'avérer intéressant dans des cas de litige entre deux matériaux.

La mise en place d'une démarche de reconnaissance automatique de matériaux par analyse colorimétrique semble quand même assez ardue, du fait de la grande 


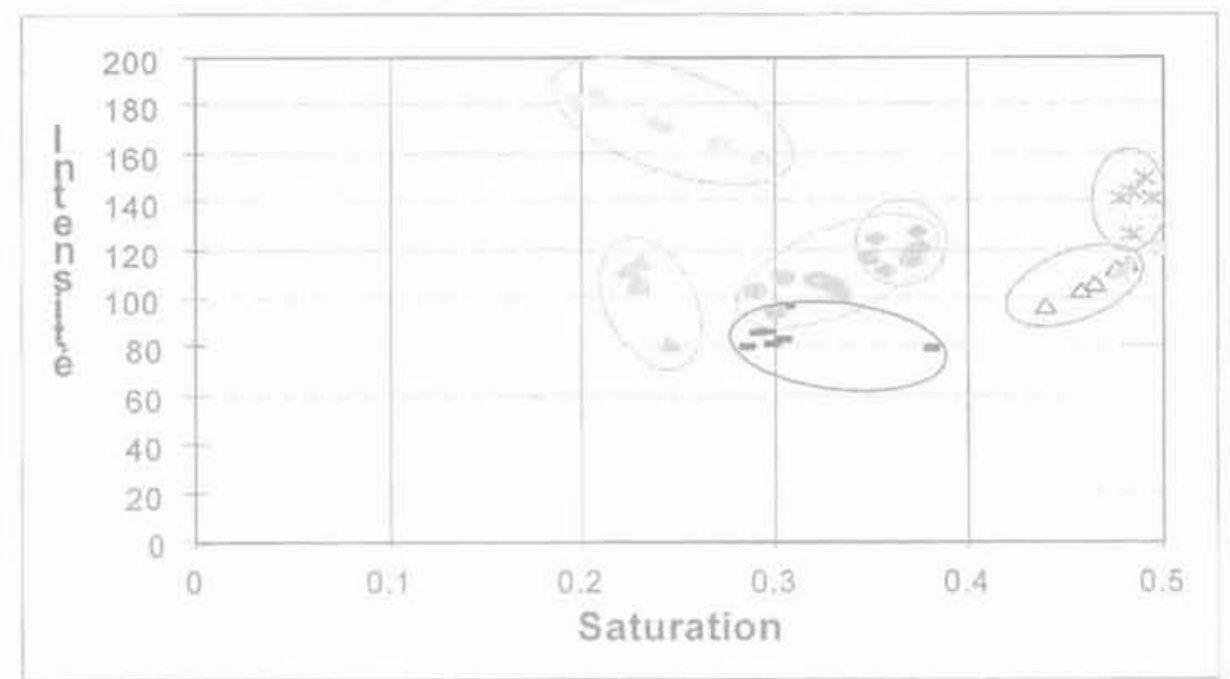

- Pouzzolane

- Argile A1

* Sablon B2 Yvelines

$\triangle$ sable B4 Essone

* sable gréseux B5 sable gris B1

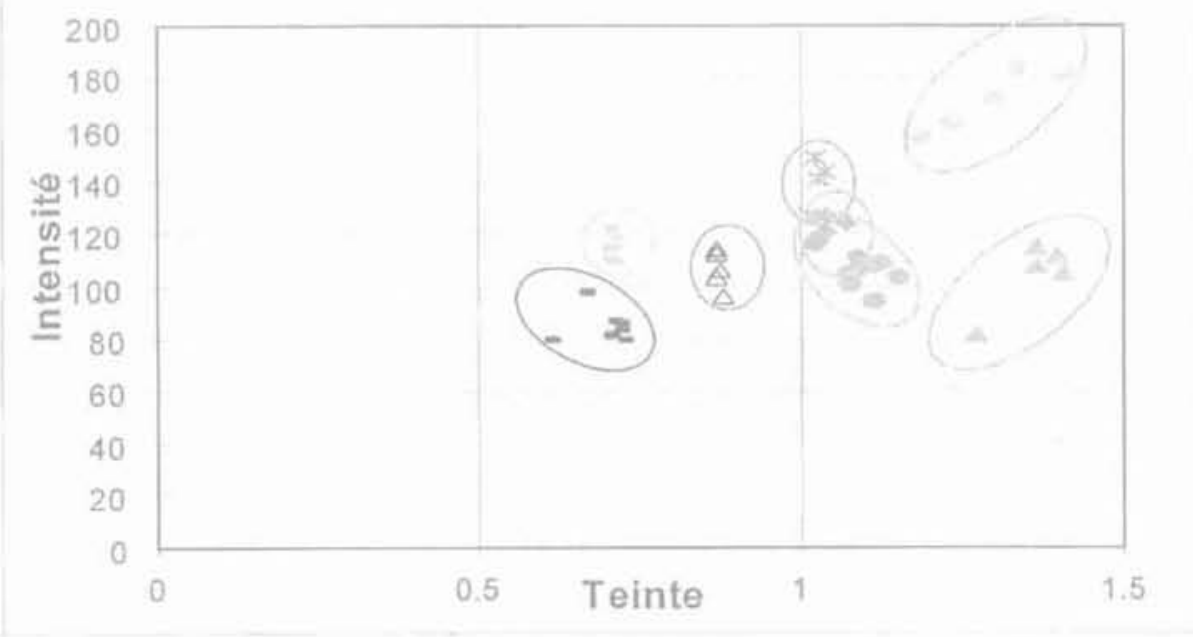

Scories A1

- Sable Allier

FG. 10 Résultat de l'analyse colorimétrique sur 8 matériaux dans le référentiel Teinte/Saturation/Intensité. Colorimetric analysis on 8 materials in the system of reference Hue/lntensity/Saturation.

variêté des types de matériaux dans la nature et de leur variation de couleur en fonction de leur composition, de la présence ou non d'impuretés et d'autres facteurs (pollution...). Mais il est possible de mettre en place pour chaque matêriau des intervalles de valeurs pour H, S et 1 caractérisant l'ídentité colorimétrique dudit matériau.

\section{5}

\section{Démarche générale d’analyse}

La démarche générale d'analyse proposêe pour caractériser les milieux granulaires en utilisant l'analyse d'images et les essais de pénétration comporte trois niveaux (Fig. 11).

Le premier niveau consiste en une reconnaissance de couches composant le milieu sondé. Cette reconnaissance permet de dissocier les matériaux non seulement du point de vue de leur nature, mais aussi de leur mise en ceuvre. Elle s'appuie sur trois analyses couplées : analyse colorimétrique, de texture et pénétrométrique. Cette analyse permet aussi de séparer parmi les différents matériaux, ceux suffisamment grenus pour permettre une analyse complémentaire de leurs caractéristiques (granulomètrie, forme...) de ceux trop fins pour pouvoir être analysés plus en détails.

Le second niveau de l'analyse permet une caracterisation physique plus précise des matériaux reconnus comme grenus à l'étape précédenté. Cette étude repose sur l'analyse granulométrique et d'angularité.

Enfin, la dernière étape de la démarche réside dans la mise en place d'une banque de données contenant, pour chaque matériau sa provenance géographique, son origine minéralogique, ainsi que pour chacune des caractéristiques physiques ou mécaniques étudiées les intervalles de variation des valeurs de chaque paramètre. Le but de cette base étant d'êtablir pour chaque matériau sa « signature physique \%, afin de faciliter une identification ou un contrồe futur. Afin de compléter cette base de données des études sur des matériaux extraits en carrières ou sur des échantillons récoltés sur site seront menées en laboratoire en conditions réelles et en faisant varier des paramètres tels que la teneur en eau ou le degré de compacité. 

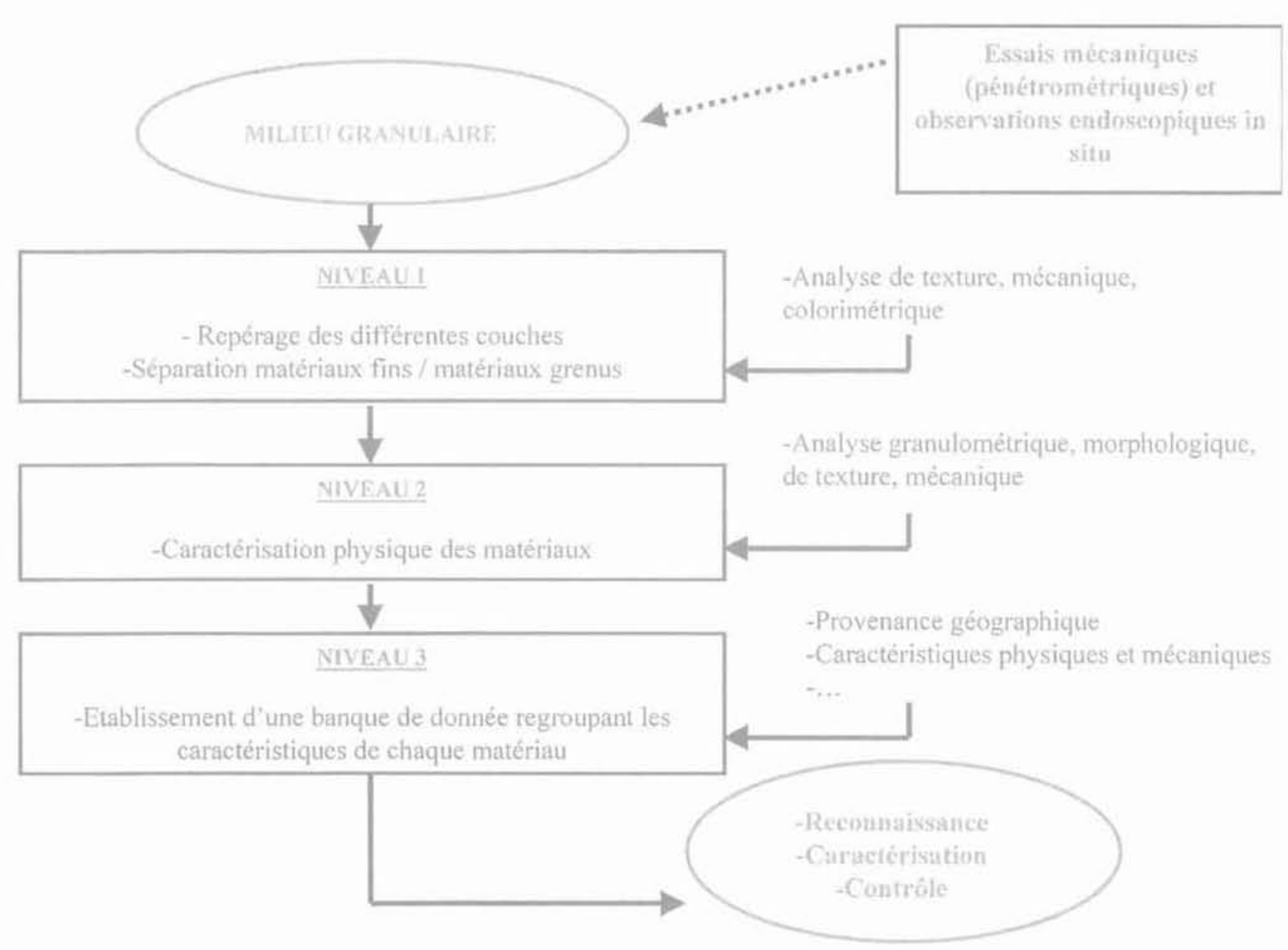

FG. 11 Démarche générale d'analyse proposée. General analysis approach.

\section{4}

\section{Intérêt du couplage de l'endoscopie avec les essais de pénétration: étude de cas}

Afin de mettre en évidence l'intérèt de l'utilisation couplée des essais de pénétration avec les essais endoscopiques est présenté maintenant un exemple d'application de cette téchnique sur un cas réel.

Cette étude a été effectuée dans le quartier de la Pardieu à Clermont-Ferrand (Breul, 1999), avant l'ouverture d'une tranchèe pour extension et modification des réseaux d'eau. Les objectifs de cette étude étaient de retrouver les différentes couches de matériaux du point de vue de leur nature et de la mise en ceuvre constituant la tranchée ainsi que la caractérisation de ces matérianx.

Le long de cette tranchée, trois essais (au niveau des reins et sur la clé de la canalisation) de pénétration dynamique, réalisés au PANDA, ont été effectués tous les $4 \mathrm{~m}$. De plus, des essais endoscopiques ont été réalisés dans certains de ces forages. Les résultats d'un des essais endoscopique et pénétrométrique, obtenus au même endroit, sont fournis à la figure 12.

L'analyse de ces résultats permet de faire plusieurs remarques. Tout d'abord, l'étude stratigraphique effectuée à partir de chaque essai, permet de constater que certaines couches du remblai sont bien mises en évi- dence sur les deux essais (couches comprises entre $0,9 / 1,2 \mathrm{~m}$ et $2,6 / 3,1 \mathrm{~m}$ ). L'analyse effectuée sur un essai peut donc ètre vérifiée et confirmée à partir de l'autre essai.

Deuxièmement, on peut noter que certaines couches de matériaux sont mises en évidence sur un des essais mais pas sur l'autre. C'est le cas entre 1,10 et $2,60 \mathrm{~m}$, où l'essai endoscopique ne repère qu' une couche (constituée donc d'un matériau identique sur toute cette hatiteuri), tandis que l'essai pénétrométrique met en évidence deux couches $(1,10 / 2,10-2,10 / 2,60)$. Cette différence s'explique par le fait qu'il s'agit d'un même matériau remblayé et compacté en deux couches. De mème, entre 0,3 et $0,9 \mathrm{~m}$, l'essai pénétrométrique permet de distinguer qư une seule couche relativement raide. L'étude de l'essai endoscopique révèle deux couches $(0,3 / 0,5$ et $0,5 / 0,9 \mathrm{~m})$ de matériaux de natures différentes (variation des paramètres d'intensité, teinte et saturation). Sur cette zone, il y a deux matériaux de nature différente mais de compacité similaire. Le couplage des deux essais permet d’avoir une complémentarité dans la caractérisation du site et d'affiner ainsi l'analyse.

Enfin, une caractérisation mème partielle des matériaux présents dans le remblai est possible à partir des essais endoscopiques. En effet, pour chaque couche mise en évidence, on a pu obtenir sa classification GTR ou au moins une idée du pourcentage de fines particules contenues dans le matériau. (mat. Grenu : matériau possédant moins de $10 \%$ de particules inférieures à $80 \mu \mathrm{m}$ ). Ces caractérisations ont été confirmées lors de l'ouver- 


\section{Moment M3 et saturation}

O $20 \quad 40 \quad B 0 \quad B 0100120140160$

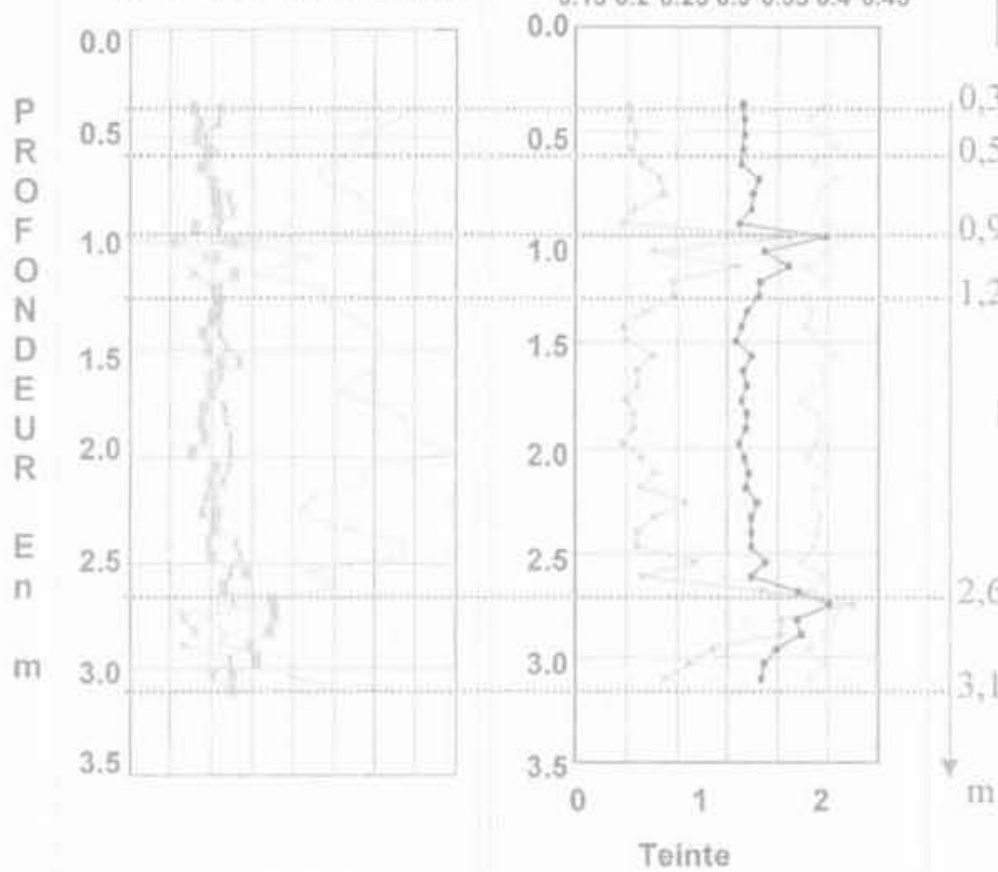

\section{Couches}

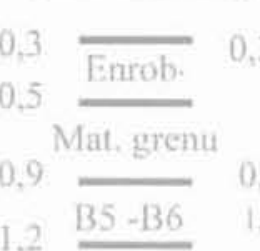

Mat. Grenu

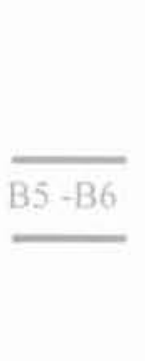

2,1

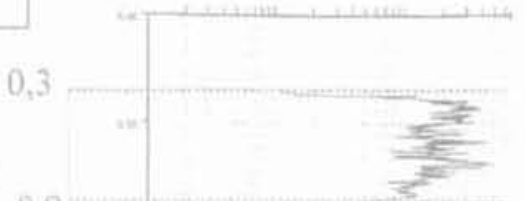

Essais endoscopiques

Essai pénétrométrique

\section{MmatMB $\rightarrow$ Saturation \\ - T-Ecart Type \\ - Coef. Variation \\ - Irtersité \\ $\rightarrow-$ Teinte}

FiG, 12 Résultat des essais endoscopiques et pénétrométriques au niveau de la clè de la canalisation. Results of endoscopic and penetration test on the pipe.

ture de la tranchée par les essais classiques de laboratoire effectués sur chaque échantillon de matériau.

\section{5}

\section{Conclusion et perspectives}

L'utilisation de l'endoscopie pour caractériser les matériaux granulaires in situ est prometteuse. Des caractéristiques, telles que la texture, la granuiométrie, l'angularité ou la couleur des matériaux peuvent déjà être analysées pour effectuer une reconnaissance de sol et/ou un contrôle de matériaux.

Son faible encombrement, sa rapidité d'intervention et le fait d'ètre peu traumatisante ouvrent à cette technique un champ d'application relativement large. Des ètudes ont déjà été menées pour le contrôle des remblais, le contrôle de la qualité de mise en cauvre des matériaux autocompactants, la reconnajssance archéologique ou la reconnaissance des neiges pour la prévention des avalanches.

De plus, le couplage de cette technique avec d'autres types d'essais (essais pénétrométriques notammentl) permet une caractérisation in situ plus complète et plus précise.

L'amẻlioration des analyses actuelles (granulométrie de tous les tvpes de matériaux), l'étude d'autres caractéristiques des milieux granulaires à partir des images (indice de concassage, forme des particules,...), de mème qu'une recherche plus approfondie sur le couplage des essais et l'estimation de la variabilité du milieu constituent les perspectives à moven terme de ce travail.

\section{Bibliographie}

Breul P. - * Caractérisation endoscopique cies milleux qrantlaires couplée â l'essai de pénetration. $\pi$ These de docteuringenieur de IUniversité de ClermontFerrand, oct. 1999, $280 \mathrm{p}$

Gourves R. - " Le PANDA: Pénètromètre dynamique léger à énergie variable pour la reconnaissance des sols, v Laboratoire LERMES, Université Blaise-Pascal. Clermont-Ferrand, 1991
SEIRA-LCPC - «GIR : Guide technique pour la réalisation des remblais et des couches de forme n (fascicules let II), 1994

Gonzalez R.C. Woods R.E. - Diqital imaqe processing. Reading, Addison-Wesley, 1992, 2nd ed., 716 p.

Raschke S.C. - ucomputer vision for expe. rimental soil micromechanics and soil characterization, s Ph. Dissertation, Univ. of Michigan, Ann Arbor, 1996, $256 \mathrm{p}$.
Coster M., Chermant I.L. - Précis d'analyse d'images. Paris, CNRS, $1985,476 \mathrm{p}$. Dordet Y. - La colorimétrie : principes et applications. Paris, Eyrolles, 1990, 149p.

Zhou S. - " Caractérisation des sols de surface à l'aide du pénétromètre dynamique lèger à ênergie variable type PANDA, is Thèse de doctorat de IT/niversité de Clermont-Ferrand, déc.1997. $180 \mathrm{p}$. 